\title{
Measuring the Added Value of Haptic Feedback
}

\author{
Emanuela Maggioni \\ SCHI Lab, School of Engineering \\ and Informatics, University of \\ Sussex, BN1 9RH Brighton, UK \\ e.maggioni@sussex.ac.uk
}

\author{
Erika Agostinelli \\ School of Engineering and \\ Informatics, University of Sussex, \\ BN1 9RH Brighton, UK \\ e.agostinelli@sussex.ac.uk
}

\author{
Marianna Obrist \\ SCHI Lab, School of Engineering \\ and Informatics, University of \\ Sussex, BN1 9RH Brighton, UK \\ m.obrist@sussex.ac.uk
}

\begin{abstract}
While there is an increased appreciation for integrating haptic feedback with audio-visual content, there is still a lack of understanding of how to quantify the added value of touch for a user's experience (UX) of multimedia content. Here we focus on three main concepts to measure this added value: UX, emotions, and expectations. We present a case study measuring the added value of haptic feedback for a standardized set of audio-visual content (i.e., short video clips), comparing two haptic stimulation modalities (i.e., mid-air vs. vibro-tactile stimuli). Our findings demonstrate that UX of hapticallyenhanced audio-visual content is perceived as a more pleasant, unpredictable, and creative experience. The users' overall liking increases together with a positive change of the users' expectations, independently from the haptic stimulation modality. We discuss how our approach provides the foundation for future work on developing a measurement model to predict the added value of haptic feedback for users' experiences within and beyond the multimedia context.
\end{abstract}

Keywords- Touch; Haptics; User Experience; Emotions; Audio-visual Content; Short Videos; Measurement; Model.

\section{INTRODUCTION}

Touch has a paramount role in enhancing the interaction between humans [1]. Indeed, touch is one of the most relevant sensorial modalities to convey emotions between humans. Similarly, touch plays an increasingly relevant role in the design of human-computer interaction (HCI). The interest in designing for tactile experiences is driven by the proliferation of haptic technologies and coupled with the desire to create more immersive, novel, and emotional engaging experiences [2]. The sense of touch is particularly explored as new interaction modality in the gaming and entertainment contexts, to enhance the experience and consumption of multimedia content and increasingly to enrich virtual reality (VR) interactions $[3,4]$. Despite the enormous potential of touch in interaction and experience design, we do lack insights into the added value of haptic stimulation on a users' experience. The question is not just simply if haptic feedback makes a difference, previous work has demonstrated positive effects already (e.g., enrich storytelling) [4], but how this difference can be quantified in relation to the user's experience?

This paper makes a contribution to this open research question. We present findings from a detailed exploration of the effect of haptic feedback on user's experience. We designed a within-participants experiment comparing two types of haptic stimulation (i.e., mid-air haptic and vibro-tactile) with a non-haptic condition for four standardized audio-visual stimuli (i.e., short video clips).

To capture participants' feedback, we combined three measures focusing on (i) user experience (UX) (hedonic and pragmatic qualities) using the AttrakDiff questionnaire [5], (ii) emotions (arousal and valence) using the Self-Assessment Manikin scale [6], and (iii) expectations (pre- and postexposure to haptic stimulation, self-reported on a 7-pont Likert scale). To account for individual differences, we also captured participants' preferences with respect to the two haptic stimulation approaches and asked for their liking of the overall experience as well as their liking for the two haptic experiences and the audio-visual content itself.

Our findings show that users' experience of audio-visual content with the addition of haptic feedback can be modified towards a more pleasant, unpredictable, and creative experience changing positively the users' expectation and the overall liking of the experience. Based on those findings, we establish an initial understanding towards the quantification of the added value of haptic feedback. This defines only the starting point for generating a measurement model that will allow predictions of users' experiences towards haptic feedback augmenting audio-visual content. Ultimately, such a model will guide content creators in their decision-making process around multisensory experience design.

\section{RELATED WORK}

This research is based on three main theoretical pillars of related work: (i) measuring UX, (ii) measuring emotions, and (iii) the role of expectations in UX. In addition, we account for the importance of user's likings and preferences affecting UX and emotions.

\section{A. Measuring UX: pragmatic and hedonic qualities}

Several attempts have been made to capture the essence of experience, and more precisely, user experience (UX) [e.g., 7, 8]. Here, we particularly build on the hedonic and pragmatic model of user experience introduced by Hassenzahl [9]. This model assumes that interactive products are perceived along two different dimensions. Pragmatic attributes relate to user's needs to achieve behavioral goals, while hedonic attributes relate to user's self [10]. Those qualities are best captured with the AttrakDiff questionnaire [11], which is a simple but effective measuring tool. The AttrakDiff ${ }^{1}$ produces quantifiable output that allows researchers and practitioners to make informed decisions along an iterative design process, either comparing one version of the product with a later version or with a competing product. The questionnaire includes 28 elements in a format of semantic differentials of opposite adjectives (e.g., unusual-ordinary, good-bad, etc.). The user can select between those word pairs using a 7-point Likert scale $(-3$

${ }^{1}$ Official website of AttrakDiff - http://www.attrakdiff.de/ 
to +3 ). Each of the 28 items is either used to rate the pragmatic quality (PQ) or the hedonic quality (HQ) and attractiveness (ATT) of an interactive product. The HQ is further divided into hedonic quality identification (HQI) and hedonic quality stimulation (HQS). Those 28 items were later on modified into a shorter version of the AttrakDiff including only 10 -items [12]. The main difference is that HQI and HQS are collapsed into a single hedonic quality scale, simplifying the repeated measurement of UX, while not losing specific insights into users' experiences.

\section{B. Measuring emotion: Arousal and valence}

As a second measurement point for our research we build on emotion research. One of the best known models for measuring emotions has suggested looking at emotions in terms of dimensions: valence (i.e., positive and negative) and arousal (i.e., high and low) [13]. This model provides a simplified view of the circumplex model [14] by just focusing on the extremes (i.e., valence and arousal axes) [14], overcoming linguistic/cultural issues and biases related to the introspective verbalization of emotions in self-report measurements [15]. The emotions dimensionality is best captured with the Self-Assessment Manikin (SAM) [6], an affective rating system, that not only includes 'valence' and 'arousal', but also a third dimension 'dominance' (the feeling of being in control or controlled). To assess those dimensions, the SAM uses graphic figures depicting the different values on the scale that indicate the emotional reactions. For the valence dimension, SAM ranges from a smiling, happy figure to a frowning, unhappy figure. For the arousal dimension, the SAM ranges from an excited, wide-eyed figure to a relaxed, sleepy figure. The dominance dimension, which is less used in UX and HCI research, ranges from a large figure (in control) to a small figure (dominated). The participant can select the appropriate visual representation and rate the experienced emotion on a 9-point rating scale for each dimension. The rating scales have been modified over the years to reflect 5and 7-point Likert scales, and hence provide a more consistent psychometric measurement.

\section{Influence of user's expectations}

In addition to measuring UX and emotions, we account for expectations as an influencer/predictor on users' experience. In fact, expectations together with beliefs play an important role in shaping our intentions, attitudes, and behaviors [16]. Expectations are socially and culturally developed attributes determining how a user would consider their behaviors/ experiences as inappropriate or appropriate in a specific context. Expectations are based on past experiences and previous knowledge. Capturing user's expectations is therefore important in order to understand emotions and users' experiences in relation to a product or service $[17,18]$.

Law et al. [8], for instance, discuss the relevance of expectations in relation to UX as follows: "If you have never used a product, we think all we can discuss is brand experience or perhaps product experience, but not user experience. Once you do interact with a product the user experience typically affects the brand experience. Everything before the first-hand encounter with a product just builds up expectations for the user experience or affects the brand experience" (p.726).
Hassenzahl and Sandweg [19] emphasized that it would be interesting to understand the formula from the expectations to different emotions and how it attributes to the overall UX. The expectations are typically measured pre and post interaction with products or services in order to understand whether the product meets the users' anticipations or if those can change after the use [20].

\section{Opportunity to measure the added value of haptics}

When it comes to measuring tactile experiences, we cannot build on specific measures, but benefit from the measures established in the field of UX and emotions research presented in the previous sections. The AttrakDiff together with the SAM provide a solid starting point for our exploration of the added value of haptic feedback, especially combining the measures of the user's expectations, personal preferences and likings.

\section{USER STUDY}

We conducted a user study to investigate the effect of haptic stimulation on audio-visual experiences. We augmented a set of short videos with either mid-air haptic or vibro-tactile stimuli and compared them to a non-haptic feedback condition. The experiment followed a within-participants design with 2 haptic devices (i.e., mid-air and vibro-tactile) x 4 videos (i.e., with different arousal and valence dimensions), and a control condition presenting the videos without haptic feedback. Each participant evaluated in total 12 stimuli.

\section{A. Haptic stimuli used}

We compared two distinct modalities of haptic stimulations: mid-air haptic stimuli and vibro-tactile stimuli.

For the mid-air haptic stimulation, we used a device developed by UltraHaptics [21]. This device uses focused ultrasound to project discrete focal points of haptic feedback on the users' hand (i.e., right hand's palm). The mid-air haptic device was positioned inside a Plexiglas box $28 \times 23 \times 17 \mathrm{~cm}$, which has a hole on the top cover to comfortably position the participants' hand with the palm $20 \mathrm{~cm}$ above the array of ultrasound speakers. The box was used to standardize the distance between the hand and the ultrasound speakers.

For the vibro-tactile stimulation, we designed a semi glove based on a $4 \times 4$ grid interface with 16 linear vibration motors (10 MM, Model: 310-117). We used a strip of fabric to create the glove and a wristband to position and safely fix the vibration motors on the participants' hand. The glove was connected to an Arduino microcontroller to control the delivery of the haptic feedback on the participants' palm. The grid was adjustable in order to deliver the vibration in the adequate locations of the palm, in function of different hand sizes.

The haptic stimuli were presented in form of a haptic pattern on the palm of the participant in each of the two haptic conditions. With patterns, we refer to a specific sequence of haptic stimuli that were projected on a participant's hand (i.e., palm). We used patterns that were previously established by Obrist et al. [22] using the above described mid-air haptic device. They identified specific design parameters (i.e., location, movement, frequency and intensity) [22] in the haptic patterns created and validated them by participants for use with standardized visual stimuli (pictures from the International 
Affective Picture System-IAPS) [23]. We used those haptic patterns. More precisely, we used the haptic patterns for low and high arousal and negative and positive valence, but excluded the neutral pattern, which was mainly used as control stimulus in the previous experiment. We also converted the haptic patterns (originally designed for mid-air) into vibrotactile stimuli based on the given design parameters in [22].

\section{B. Audio-visual stimuli used}

To ensure consistency between the haptic patterns and the audio-visual stimuli, we used the same thematic stimuli for the selection of the audio-visual stimuli (i.e., short videos). We searched for appropriate video clips on YouTube, which we cut into 6-second videos, following the length used in the International Affective Digitalized Sounds (IADS) library, and then we identified relevant audio files from this IADS library [24] to be added to the videos. Thus, we aimed to ensure that both visual and audio are standardized with respect to their ability to convey specific emotions.

The following themes were represented in the final audiovisual stimuli (see Tab. 1) (please note we cannot include the visuals for the IAPS pictures due to copyright reason, but we provide the picture and sound number \# as reference):

1. Graveyard scenery: representing low arousal and negative valence video, chosen based on the IAPS picture $\# 9001$, with the addition of a slow piano sound track sourced from YouTube as there was no appropriate sound in the IADS library.

2. Calm forest scenery: representing low arousal and positive valence, chosen based on the IAPS picture \#5201, with the addition of a singing bird audio track from IADS, sound number \#151.

3. Burning house scene: representing high arousal and negative valence, chosen based on the IAPS picture \#8485, with the addition of a scared screaming man audio track from IADS, sound number \#292.

4. Rafting scene: representing high arousal and positive valence), chosen based on the IAPS image number $\# 8370$, with the addition of joyful screaming guys' audio track sourced from YouTube as there was no appropriate sound in the IADS library.

\begin{tabular}{|l|c|c|}
\hline \multirow{2}{*}{ Audio-Visual Stimuli } & \multicolumn{2}{|c|}{ Emotion Dimensions } \\
\cline { 2 - 3 } & Arousal & Valence \\
\hline 1) Graveyard scenery & Low & Negative \\
\hline 2) Calm forest scenery & Low & Positive \\
\hline 3) Burning house scene & High & Negative \\
\hline 4) Rafting scene & High & Positive \\
\hline
\end{tabular}

TABLE 1. The four audio-visual stimuli used in the study.

We evaluated the four audio-visual stimuli to ensure the content congruency between the visual scene and audio. Twenty participants were instructed to watch the videos with headphones and to rate valence and arousal for each stimulus using the 7-point Likert scale SAM questionnaire [6]. The results confirm on average a good mapping of each video towards the intended affective rating (statistically significant difference between videos, $F(3,57)=31.60, p<0.001)$.

\section{Measures used}

The measurements used to investigate the added value of haptic feedback are summarized below.

\section{1) AttrakDiff questionnaire}

In our study, we used the short version of the AttrakDiff questionnaire including 10 items, as it is more suitable for repeated measures and at the same time still allows to capture the users' experience (details in the related work section). The questionnaire is composed of the following three main factors:

Pragmatic qualities (PQ, $\alpha=0.719), 4$ items; referring to the usability of the haptic feedback.

Hedonic qualities (HQ, $\alpha=0.867), 4$ items composed by 2 items measuring HQ Identification (HQI, $\alpha=0.783$ ) and 2 items measuring HQ Stimulation (HQS, $\alpha=0.811$ ); referring to the hedonic attributes relate to the user's self.

Attractiveness of the product (ATT, $\alpha=0.874$ ), 2 items referring to the aesthetic elements of the haptic stimulation.

\section{2) SAM affective scale}

In order to measure the emotions associated with the haptically-enhanced audio-visual content we used the SAM scale for each haptic stimulation modality: mid-air and vibrotactile stimulation. We can capture the emotional reaction based on the valence and arousal ratings on a 7-point Likert scale. The dominance dimension was not used in our study as it was a passive rather than active interaction scenario and hence not meaningful in our experimental set-up. This enabled us to compare the added value for the two different haptic stimulation modalities.

\section{3) Expectation scale}

Users expectations towards the two haptic devices were captured through two items:

[i] Comfort with the haptic feedback (mid-air and vibrotactile stimulation), rating the following question: "I think the haptic feedback will be comfortable while watching a video" on a 7 point-Likert scale, where 1 was not comfortable at all and 7 was very comfortable. This item was considered relevant with respect to the user's need for comfort when experiencing haptic feedback.

[ii] Ability of the haptic device to convey emotions, rating the following question: "I think the haptic feedback is able to convey emotions" on a 7 point-Likert scale, where 1 was not possible at all and 7 was very possible. This item was considered relevant with respect to the assumption that haptic feedback has a positive effect on user's emotional engagement shown in prior research [2]. Those questions were asked before the study start (no familiarization, only visual appearance of the device was evaluated). The same two items were evaluated again post experiment, asking the following questions: "I felt comfortable while watching the video" and "I think the haptic feedback conveyed emotions while watching the video".

\section{4) Liking scores and preferences}

In addition to the above measures, we also used Liking scores as a self-report measure to evaluate the users overall experience (captured on a 7-point Likert scale). The liking was measured for each component composing the experience: the 
mid-air versus vibro-tactile experience and the audio-visual content itself. Moreover, we also captured the user's preferences for the two haptic stimulation modalities compared in our study by asking the following questions: "I enjoyed using mid-air haptic device" and "I enjoyed using vibro-tactile device" (on a 7-point Likert scale).

\section{Procedure}

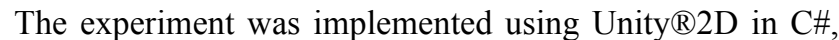
enabling participants to follow the instructions shown on a 24 " computer screen in front of them and instruction provided through headphones. Participants were initially instructed to answer the socio-demographic and expectation questions on the screen. Afterwards, all four audio-visual stimuli were presented in randomized order to the participant, either without or with haptic feedback. The haptic stimuli were randomized in blocks. Participants either experienced all the mid-air haptic stimuli first or all the vibro-tactile stimuli. The order of the audio-visual stimuli was randomized for each block and participant. After each stimulus, participants were presented with the AttrakDiff questionnaire, SAM, and the liking scale. In the last part of the experiment, participants rated the overall liking of the experience with the two different haptic feedback modalities and completed once more the expectations questions accounting for the perceived comfort and assessing the device's ability to convey emotions through haptic feedback. Finally, the participants' preference for one or the other type of haptic feedback was captured. All participants expressed written consent before starting the experiment. The study was approved by the local Ethics Committee.

\section{E. Participants}

We recruited sixteen right-handed participants for the experiment $\left(M_{\text {age }}=30, S D=4\right), 10$ females. The whole experiment lasted about 45 minutes and each participant was exposed to all 3 conditions in a randomized order: a) audiovisual stimulation only, b) audio-visual stimulation with vibrotactile haptic feedback, and c) audio-visual stimulation with mid-air haptic feedback.

\section{RESULTS}

Here we present the results from our study, focusing first on the expectations (pre-post) and then on the Liking scores and preferences expressed by participants. We combined together the haptic conditions or presented them separate when meaningful or significant for the analyses. Based on this we will then discuss the UX and emotion results.

\section{A. Expectations for haptic stimulation}

Participants ratings on the expectations of the haptic feedback to convey emotions generally changed from pre- to postexposure $(F(1,15)=8.758, p<0.01)$, with the average score increasing in the post-ratings for both haptic conditions (see Fig.1). We found no difference between the two haptic stimulation modalities. Similarly, the expectations regarding the comfort of the overall experience (haptically-enhanced audio-visual content) were significantly different between the pre- and post-exposure $(F(1,15)=12.739, p<0.01)$. As before with an average score that increased in the post stimulation (see Fig.1) but with no difference between the two haptic feedback modalities.

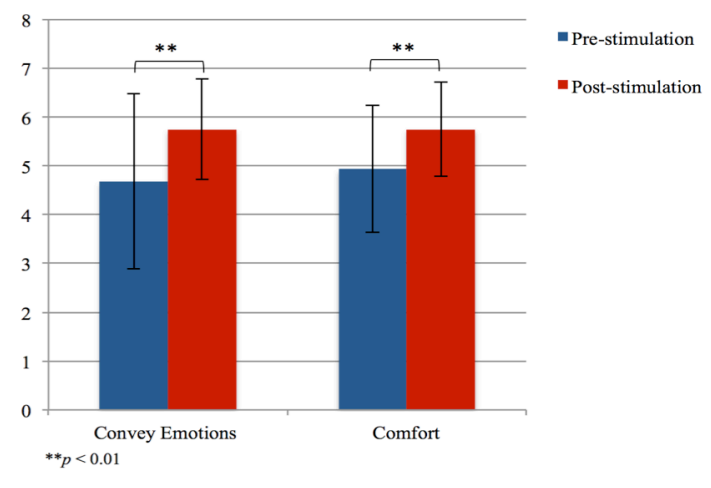

Fig. 1. The mean participants'expectations pre-post stimulation concerning the capacity of both haptic devices to convey emotions and the comfort of the overall experience, with no distinction between devices. Asterisks represent significant differences at the level of $p<0.01$. Error bars, \pm standard deviations $(S D)$.

\section{B. Likings and preferences for haptic feedback}

With respect to users' preferences for a haptic technology: mid-air vs. vibro-tactile stimulation, our results using ANOVA suggest $(F(1,15)=13.96, p<0.01)$ that the participants preferred the vibro-tactile stimulation $(M=6.19, S D=0.834)$ over the mid-air stimulation $(M=5.06, S D=1.48)$. Three elements were considered in analyzing the liking scores: (1) the overall liking of the experience, (2) the liking of the haptic feedback (both conditions), (3) the liking of the audio-visual stimuli. We performed the following mixed model:

$$
\mathrm{OL}=\mathrm{G}_{\mathrm{i}}+\mathrm{C}_{\mathrm{k}}+\mathrm{LH}_{\mathrm{ijk}}+\mathrm{LV}_{\mathrm{ijk}}+\mathrm{ME}_{\mathrm{i}}+\mathrm{VE}_{\mathrm{i}}+\operatorname{Vid}_{\mathrm{j}} \varepsilon_{\mathrm{ij}}(*),
$$
where $\mathrm{OL}$ is the Overall Liking, $\mathrm{G}$ is participant's gender, $\mathrm{C}$ is the condition, $\mathrm{LH}$ is the liking of the haptic feedback and LV is the liking of the video, $\mathrm{ME}$ is rating of the experience with mid-air technology and VE is the rating of the experience with the vibro-tactile technology, Vid is the video. Age has not been included due to the homogeneity in age of the sample.

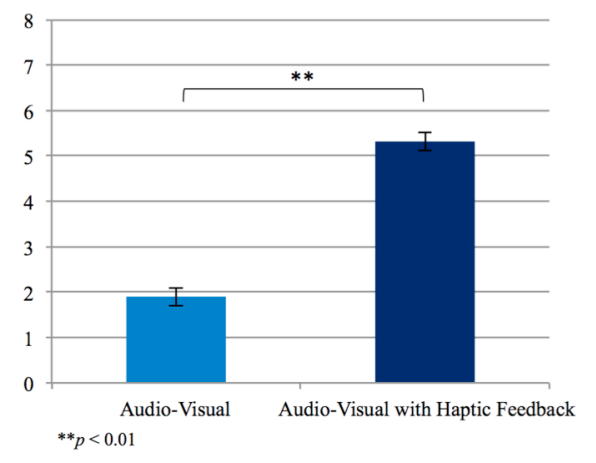

Fig. 2. The mean participants' Overal Liking of the experience for audiovisual content and for haptically-enhanced audio-visual content. Asterisks represent significant differences at the level of $p<0.01$. Error bars, $\pm S D$.

The random variable in the model is the video. The model shows a statistically significant difference in the conditions with haptic feedback, without differences between the devices. T-test pairwise comparisons show a statistically significant difference between the condition without and with haptic 
feedback in the OL $(\mathrm{t}(1)=10.66, \mathrm{p}<0.001)$ (see Fig. 2). We did not find an effect of gender on the OL score.

\section{User's experiences}

To analyze the AttrakDiff results we performed the following mixed model for each questionnaire item:

$V_{w}=C_{k}+I D_{i}+\varepsilon_{i k}(*)$, where the $\mathrm{V}$ is the questionnaire item, $\mathrm{C}$ is the condition and ID is the participant identification number that represents the random effect, which is due to the participants' idiosyncrasies during the rating process. Finally, $\varepsilon$ is the random error of the model. The model, with a Bonferroni adjustment pairwise comparisons of each condition (see Fig. 3), shows a statistical difference on the item 'unpredictable-predictable' between the audio-visual and the vibro-tactile stimulation condition $(t(300)=2.84, p<0.01)$ and barely significant between the audio-visual and the mid-hair stimulation condition $(t(300)=2.37, p=0.05)$. Similar results are obtained for the item 'complicated-simple' in the audiovisual condition is different from the vibro-tactile stimulation condition $(t(300)=2.80, p<0.05)$, the item 'cheap-premium' in audio-visual condition is different from the vibro-tactile stimulation condition $(t(300)=-2.59, p<0.05)$, and the item 'unimaginative-creative' shows similar results between the audio-visual and the vibro-tactile stimulation condition $(t(300)=-3.45, p<0.01)$.

The results show a difference between the participants' experiences with and without haptic feedback on both dimensions: the pragmatic (PQ) and hedonic qualities (HQ). In particular, concerning the PQ the vibro-tactile device and the mid-air device are generally experienced as unpredictable, the vibro-tactile device is also perceived as more complicated in comparison with the only audio-visual experience. With respect to the HQ, the vibro-tactile device is experienced as premium (more expensive) and more creative, in comparison with the only audio-visual experience.

\section{Emotion ratings}

To analyze the data collected through the SAM scale on valence and arousal ratings we performed the following mixed model, considering arousal (A) and valence (V) separately:

A or $V=G_{i}+C_{j}+A e_{i}+I_{i}+\varepsilon_{i j}(*)$, where $G$ is the gender of the participant, $\mathrm{C}$ is the condition, the participants' age, ID is the random effect due to the participants' idiosyncrasies, $\varepsilon$ is the random error of the model. We found no statistically relevant differences between the haptic feedback conditions and the audio-visual stimuli.

\section{DISCUSSION AND CONCLUSIONS}

This paper's ambition was to measure the added value of haptic feedback when integrated with audio-visual content (i.e., short video clips). We used standardized audio-visual and haptic stimuli combining established measurements to capture users' experiences and emotions, extended with additional measures on users' expectations, individual likings, and preferences regarding the haptic stimuli used.

"Legend: ${ }_{\mathrm{w}}=1-10$, number of the questionnaire items; ${ }_{\mathrm{k}}=1-3$, number of conditions; ${ }_{i}=1-16$, number of participants $; j=1-4$, number of the videos.

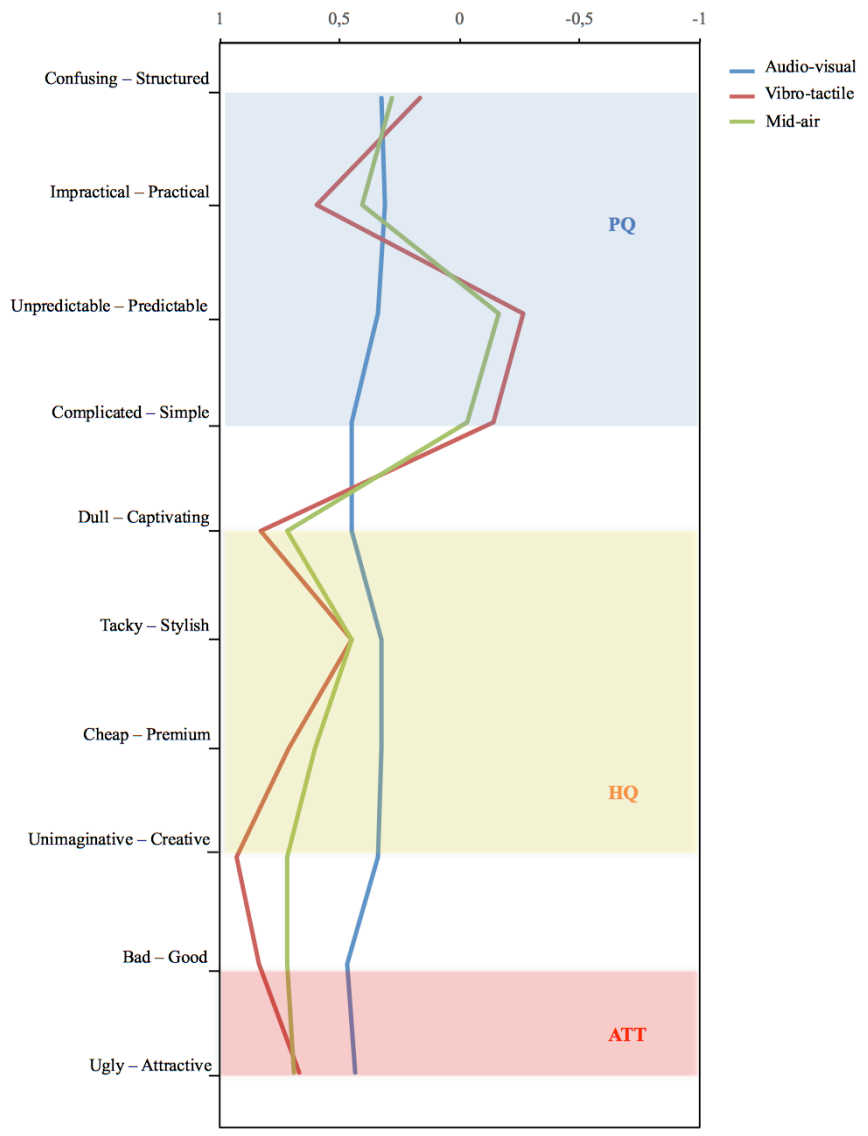

Fig. 3. The mean participants' AttrakDiff scores in the three different conditions (i.e., blue line for audio-visual content, red line for audio-visual content haptically- enhanced with vibro-tactile feedback, green line for audiovisual content haptically-enhanched with mid-air feedback): PQ refers to the Pragmatic qualities, HQ to the Hedonic qualities, and ATT to the Attractiveness of the products.

The findings provide a promising starting point towards the quantification of the added value of haptic feedback, but do not allow a clear distinction between the different types of haptic feedback, with respect to the emotions ratings. However, our findings clearly suggest that the haptic feedback, independently from the stimulation modality used, enhances the users experience, confirming previous research $[2,3,4]$. With respect to the two stimulation modalities, our findings show an increase in participants' ratings from pre- and post-exposure, with a preference for the vibro-tactile feedback. This could be explained by the user's familiarity with vibro-tactile feedback in contrast to mid-air haptic feedback, together with its presentation in form of a semi glove, fitting the hands' ergonomics instead of placing the hand on top of a box. However, the comfort ratings and ratings for each devices' ability to convey emotions increased from pre- to postexposure, with no difference between devices.

The self-report results show how the users' expectations towards haptic stimulation positively changed after exposure, which is promising, and underlines the relevance to capture user's expectations in the evaluation of products and interactions [17, 18]. The overall liking results show an increment of the general pleasantness of the experience 
enhanced through haptic feedback, without any major distinction between mid-air and vibro-tactile devices.

While this study provides a promising starting point towards the ambition to create a measurement model to quantify the added value of touch for multimedia experiences, we also acknowledge the limitations of the current study. First, the sample size is too small to allow general conclusions on the three different conditions. Hence, our findings need to be further validated, introducing additionally the measurement of the user' fatigue. The prolonged use of the haptic device could in fact induce fatigue in the user, impacting the overall experience [25]. Second, the number of audio-visual stimuli needs to be increased and tested in different combinations of congruent and incongruent haptic feedback. Such extension would allow to move beyond the content format (short videos) and standardized stimuli (from the IAPS and IADS library) towards full-length movies, which would increase the ecological validity of our measurement approach. Finally, and more for future work, it is worth to extend the measurement approach towards physiological measures including electroencephalograms (EEG) [26] and galvanic skin responses (GSR) [27] that are increasingly used for UX and emotion studies in HCI.

We hope that our research will inspire future research into the quantification of the added value of haptic feedback for a variety of content formats and haptic devices. That will allow the creation of a structural equation model to predict and estimate the added value of haptics for user experiences. Consequently, it will extend the quality of experience (QoE) research [28] for multimedia applications, but also guide the design decisions in other application contexts, such as gaming and virtual reality experiences.

\section{ACKNOWLEDGMENTS}

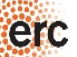

This project has received funding from the European Research council (ERC) under the European Union's Horizon 2020 research and innovation programme, grant agreement 638605.

\section{REFERENCES}

[1] M. J. Hertenstein, R. Holmes, M. McCullough, and D. Keltner, "The communication of emotion via touch." Emotion, 9.4, 2009, pp. 566- 573.

[2] O. S. Schneider, and K. E. MacLean, "Improvising design with a haptic instrument." In Haptics Symposium (HAPTICS), 2014 IEEE, pp. 327332.

[3] M. Azmandian, M. Hancock, H. Benko, E. Ofek, and A. D. Wilson, "Haptic retargeting video showcase: dynamic repurposing of passive haptics for enhanced virtual reality experience." Proceedings of the 2016 CHI Conference Extended Abstracts on Human Factors in Computing Systems. ACM, 2016, pp. 3-3.

[4] A. Israr, S. Zhao, K. Schwalje, R. Klatzky, and J. Lehman, "Feel effects: enriching storytelling with haptic feedback." ACM Transactions on Applied Perception (TAP), 11.3, 2014, pp. 11-17.

[5] M. Hassenzahl, M. Burmester, and F. Koller, "AttrakDiff: A questionnaire for measuring perceived hedonic and pragmatic quality". [Ein Fragebogen zur Messung wahrgenommener hedonischer und pragmatischer Qualität]. In J. Ziegler and G. Szwillus (Eds.) Mensch \& Computer. Interaktion in Bewegung, B.G. Teubner, 2003, pp. 187-196.
[6] M. M. Bradley and P. J. Lang, "Measuring emotion: the self-assessment manikin and the semantic differential." Journal of Behavior Therapy and Experimental Psychiatry, 25.1, 1994, pp. 49-59

[7] M. Hassenzahl and N. Tractinsky, "User Experience - a research agenda" [Editorial]. Behavior \& Information Tech., 25, 2006, pp. 91-97.

[8] E. L. C Law, V. Roto, M. Hassenzahl, A. P. Vermeeren and J. Kort, "Understanding, scoping and defining user experience: a survey approach." In Proceedings of the SIGCHI conference on human factors in computing systems, ACM, 2009, pp. 719-728

[9] M. Hassenzahl, "The hedonic/pragmatic model of user experience." Towards a UX Manifesto, 2007, pp. 10-14.

[10] M. Hassenzahl, "The thing and I: Understanding the relationship between user and product.” In Funology, Springer, 2003, pp. 31-42.

[11] M. Hassenzahl and A. Monk, "The inference of perceived usability from beauty," Human - Computer Interaction, 25.3, 2016, pp. 253-260.

[12] M. Hassenzahl, S. Diefenbach, and A. Göritz, "Interacting with Computers needs, affect, and interactive products - Facets of user experience.” Interact. Comput., 2010, 22.5, pp. 353-362.

[13] P. J. Lang, "The emotion probe: Studies of motivation and attention." American Psychologist, 50.5, 1995, pp. 372-385.

[14] L.F. Barrett and J. A. Russell, "Independence and bipolarity in the structure of current affect." Journal of Personality and Social Psychology, 74.4, 1998, pp. 967-984.

[15] R. W. Levenson, P. Ekman, K. Heider, and W.V. Friesen, "Emotion and autonomic nervous system activity in the Minangkabau of West Sumatra.” J. of Personality and Social Psy., 62.6, 1992, pp. 972-988.

[16] M. Fishbein and I. Ajzen, "Predicting and changing behavior: The reasoned action approach.” Taylor \& Francis, 2011.

[17] E. Karapanos, "User experience over time." Modeling Users' Experiences with Interactive Systems, Springer Berlin Heidelberg, 2013, pp. $57-83$.

[18] L. Gegner and R. Mikael, "For what it is worth - Anticipated experience evaluation." In 8th International Conference on Design and Emotion, London, UK, 2012, pp. 11-14.

[19] M. Hassenzahl, and N. Sandweg, "From mental effort to perceived usability: Transforming experiences into summary assessments." Proceedings of ACM CHI, Extended Abstracts, 2004, pp. 1283-1286.

[20] J. Noyes, "Expectations and their forgotten role in hci." In Encyclopedia of Human Computer Interaction, IGI Global, 2006, pp. 205-210.

[21] T. Carter, S. A. Seah, B. Long, B. Drinkwater, and S. Subramanian, "ultrahaptics: multi-point mid-air haptic feedback for touch surfaces." Proc. 26th Annu. ACM Symp. UI Softw. Technol., 2013, pp. 505-514.

[22] M. Obrist, S. Subramanian, E. Gatti, B. Long, and T. Carter, "Emotions Mediated Through Mid-Air Haptics." Proc. 33rd Annu. ACM Conf. Hum. Factors Comput. Syst., 2015, pp. 2053-2062.

[23] P. J Lang, M. M. Bradley, and B. N. Cuthbert, "International affective picture system (IAPS): Affective ratings of pictures and instruction manual." Technical Report A-6, Gainesville, FL:Univ. of Florida, 2005.

[24] M. M. Bradley and P. J. Lang, "International affective digitized sounds (IADS): Stimuli, instruction manual and affective ratings", Tech. Rep. No. B-2. Gainesville, FL: The Center for Research in Psychophysiology, University of Florida, 1999.

[25] A. Hamam and A. El Saddik, "Toward a mathematical model for quality of experience evaluation of haptic applications." In IEEE Transactions on Instrumentation and Measurement, 2013, 62.12, pp. 3315-3322.

[26] A. Moldovan, I. Ghergulescu, S. Weibelzahl, and C. H. Muntean, "Usercentered EEG-based Multimedia Quality Assessment." Broadband Multimedia Systems and Broadcasting (BMSB), IEEE International Symposium on IEEE, 2013, pp. 1-8.

[27] R. L. Mandryk, K. M. Inkpen, and T. W. Calvert, "Using psychophysiological techniques to measure user experience with entertainment technologies." Behav. Inf. Technol., 3001, 2006, pp. 141158.

[28] I. Wechsung and K. De Moor, "Quality of experience versus user experience." In S. Möller and A. Raake, Quality of Experience, Springer International Publishing, 2014, pp. 35-54. 